(c) 2021 Universidad Nacional Autónoma de México, Facultad de Estudios Superiores Zaragoza.

Este es un artículo Open Access bajo la licencia CC BY-NC-ND (http://creativecommons.org/licenses/by-nc-nd/4.0/).

TIP Revista Especializada en Ciencias Químico-Biológicas, 24: 1-12, 2021.

https://doi.org/10.22201/fesz.23958723e.2021.373

\title{
Modelos animales en el estudio del síndrome metabólico
}

\author{
José Rene Escalona Mugica ${ }^{1}$, Antonio Barajas Martínez², Orlando A. Alfaro Becerril ${ }^{3}$, \\ Francisco Estrada Rojo ${ }^{2}$, Manuel Ángeles Castellanos ${ }^{3}$ y Laura M. Ubaldo-Reyes ${ }^{3 *}$ \\ ${ }^{1}$ Departamento de Embriología y Genética, ${ }^{2}$ Departamento de Fisiología, ${ }^{3}$ Departamento de Anatomía, \\ Facultad de Medicina, Universidad Nacional Autónoma de México, Av. Universidad \# 3000, \\ Alcaldía Coyoacán 04510, Ciudad de México, México. E-mail:*lm.ubaldo@facmed.unam.mx
}

\begin{abstract}
RESUMEN
El término síndrome metabólico se refiere a una serie de factores de riesgo que resultan de un desbalance metabólico. Se han señalado varias causas para su desarrollo, entre las que destacan la ingesta excesiva de calorías y el sedentarismo. Este desbalance entre la ingesta y el gasto energético resulta en un incremento de peso en la forma de tejido adiposo, estrechamente asociado con múltiples desórdenes metabólicos. El síndrome metabólico, así como sus consecuencias, representan un serio problema de salud pública a nivel mundial. De ahí, surge la importancia del establecimiento de estrategias exitosas para su diagnóstico y tratamiento. Si bien los estudios epidemiológicos arrojan bastante información, por razones éticas y metodológicas, es necesario abordar los aspectos experimentales con modelos animales. A la fecha, existen múltiples modelos de manera que la elección de uno en específico requiere de la cuidadosa consideración de las variables o fenómenos a estudiar. En esta revisión se abordan aspectos generales del síndrome metabólico. Asimismo, se discuten las características generales de los modelos murinos más empleados en su estudio, como son los basados en dietas altas en carbohidratos y en grasas, además de los genéticos. En particular, para los modelos de dietas altas en grasa, se consideran otros aspectos como el porcentaje de kcal provenientes de la grasa, el tipo de ácidos grasos empleados, los regímenes de alimentación, así como los efectos multigeneracionales.
\end{abstract}

Palabras clave: síndrome metabólico, obesidad, dieta, carbohidratos, grasa, modelos animales.

\section{Animal models in the study of metabolic syndrome}

\begin{abstract}
The term metabolic syndrome refers to a series of risk factors that lead to a metabolic imbalance. There are various causes in the development of metabolic syndrome, among the most prevalent are excessive calorie intake and low physical activity. The resulting imbalance between energy intake and expenditure leads to weigh gain in the form of adipose tissue, which is tightly linked to multiple metabolic anomalies. Metabolic syndrome and its consequences are a public health concern worldwide. Although epidemiologic studies provide ample information regarding the pathogenesis of metabolic syndrome, ethical and methodological concerns make research on animal models necessary. The choice of a particular model requires the careful analysis of the variables or phenomenon to be studied, as multiple animal models of metabolic syndrome are currently available. This review covers general elements of metabolic syndrome. In addition, we discuss basic aspects of the most common murine models, taking into account models induced by high-sugar diets, high-fat diets and genetic models. Particularly for high-fat diet models, other aspects are considered, such as the percentage of kcal from fat, the type of fatty acids included in the diet, as well as multigenerational effects.
\end{abstract}

Keywords: metabolic syndrome, obesity, diet, carbohydrates, fat, animal models. 


\section{INTRODUCCIÓN}

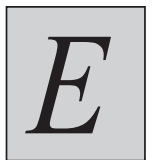

1 síndrome metabólico (SM) es una condición que afecta sin distinción a individuos de cualquier país y estrato socioeconómico. Entre los factores de riesgo identificados para su desarrollo se encuentran: la obesidad, la hiperglucemia, la dislipidemia y la hipertensión. El SM no es un desorden moderno, las condiciones clínicas asociadas al sobrepeso ya eran reconocidas por Dickens en 1836 a través de su personaje Pickwick. El autor describe los rudimentos de lo que hoy se conoce como síndrome de hipoventilación por obesidad. Sin embargo, la primera descripción formal de la coalescencia entre los elementos que actualmente constituyen el SM se debe al anatomista Morgagni, 1861 quien en su obra “DeSedibus et Causis Morborum per Anatomen Indagata"(Los fundamentos y causas de las enfermedades investigadas por la anatomía) y citado por (Enzi, Busetto, Inelmen, Coin, \& Sergi, 2003) relata dos casos en donde coexisten la obesidad visceral, hipertensión, hiperuricemia, ateroesclerosis y apnea obstructiva. Durante el siglo XIX, los diagnósticos eran entidades claras descritas como enfermedades manifiestas de forma anatómica o clínica que llamaban la atención por lo infrecuentes que eran en la población general.

En ese mismo siglo, un descubrimiento posibilitaría la creación posterior del concepto SM: el índice de Quetelet, 1832 descrito con fines estadísticos para predecir la mortalidad; después fue rebautizado como Índice de Masa Corporal (IMC) por Ancel Keys, 1972 ambos autores citados por (Eknoyan, 2007). Esto implicó dos puntos cruciales: primero, la intención estadística de relacionar las condiciones actuales de un individuo para predecir su mortalidad en el futuro y segundo, aclarar que el peso no es del todo malo por sí mismo, sino la pérdida de su equilibrio en relación con la estatura.

En el siglo XX, desde 1920 hasta 1980 se formularon numerosas descripciones precursoras del SM. En 1923 Eski Kylin describió una relación entre hipertensión, gota e hiperglucemia (Rössner, 2009). Mientras que en 1956, el Dr. Jean Vague publicó la asociación entre aterosclerosis, diabetes, gota y cálculos renales con la obesidad central (Vague, 1956). Posteriormente el término SM fue acuñado por Singery Haller cuando estudiaban pacientes con dislipidemia, encontraron la manifestación de enfermedades como la diabetes, la obesidad, la gota, el hígado graso y la hiperlipoproteinemia. Más tarde, Singer agregó la hipertensión (Haller, 1977). Simultáneamente, durante este siglo ocurrieron avances técnicos importantes, el desarrollo de la técnica de medición de la presión arterial mediante los ruidos auscultatorios de Korotkoff (Booth, 1977), la identificación de las fracciones de colesterol unidas a las lipoproteínas de alta densidad (HDL) (Schaefer et al., 2000) y la detección de la insulina por medio de radioinmunoensayo (Aronis \& Mantzoros, 2012). Finalmente, estos hallazgos bioquímicos fueron complementados con el estudio de la cohorte Frahmingham, que permitió relacionarlos con el riesgo cardiovascular (Dawber, Moore, \& Mann, 1957).
Es en este contexto que se logró gestar finalmente la definición de SM de Reaven como el resultado de una fisiopatología común, la resistencia a la acción de la insulina (Reaven, 1988).

Una tercera etapa de definiciones operacionales siguió con rapidez a la de Reaven. A partir de la primera definición de la Organización Mundial de la Salud (OMS) en 1998 (K. G. Alberti \& Zimmet, 1998) diversas organizaciones intentaron utilizar el concepto de SM no como una asociación de factores entre enfermedades, sino como una herramienta de diagnóstico temprano antes del desarrollo de la diabetes o de una enfermedad cardiovascular. Con este fin, las definiciones operacionales se construyeron primero utilizando los valores obtenidos a través de los diagnósticos de diversas enfermedades. Durante el cambio de milenio estos valores buscaron un giro preventivo, al disminuir su umbral e incrementar su sensibilidad. En la Tabla I se detallan los cambios que han tenido los criterios para el diagnóstico del SM a través del tiempo. En este sentido, comienzan a aparecer los conceptos de: prediabetes, presión arterial elevada y sobrepeso. Actualmente, hay descritas más de 160 comorbilidades asociadas al sobrepeso y una cantidad creciente de biomarcadores asociados (O'Neill, Bohl, Gregersen, Hermansen, \& O’Driscoll, 2016).

El diagnóstico temprano y oportuno del SM en la población aparentemente sana, permite establecer medidas de prevención para el desarrollo de otras comorbilidades, así como la progresión de enfermedades crónico-degenerativas y una mala calidad de vida. La OMS reporta 38 millones de muertes al año atribuidas a las enfermedades no transmisibles (ENT). El $75 \%$ de muertes anuales en el mundo se debe a causa de las ENT, 3 de cada 5 muertes son causadas por esas patologías, principalmente en los países de ingresos bajos a medios (K. G. M. M. Alberti,Zimmet, $\&$ Shaw, 2006). Entre las causas de mortalidad a nivel mundial, anualmente 17.5 millones son atribuibles a las enfermedades cardiovasculares (EVC) y 1.5 millones a la diabetes mellitus 2 (DM2). La presencia del SM incrementa 5 veces el riesgo de desarrollar DM2 y 3 veces el riesgo de EVC (Saklayen, 2018).

\section{VARIACIÓN DE LOS CRITERIOS DIAGNÓSTICOS}

Las últimas definiciones de SM han puesto énfasis en el uso de criterios específicos para cada población, particularmente en el aspecto de la circunferencia abdominal. Los criterios para el diagnóstico del SM según las recomendaciones de las guías de la Adult Treatment Panel III (ATP-III) ("Third Report of the National Cholesterol Education Program (NCEP) Expert Panel on Detection, Evaluation, and Treatment of High Blood Cholesterol in Adults (Adult Treatment Panel III) Final Report”, 2002), son la presencia de al menos 3 de los siguientes padecimientos:

1. Obesidad abdominal: un perímetro de cintura $\geq 102 \mathrm{~cm}$ en hombres $\mathrm{y} \geq 88 \mathrm{~cm}$ en mujeres.

2. Triacilgliceroles elevados: concentraciones $>150 \mathrm{mg} / \mathrm{dL}$ 


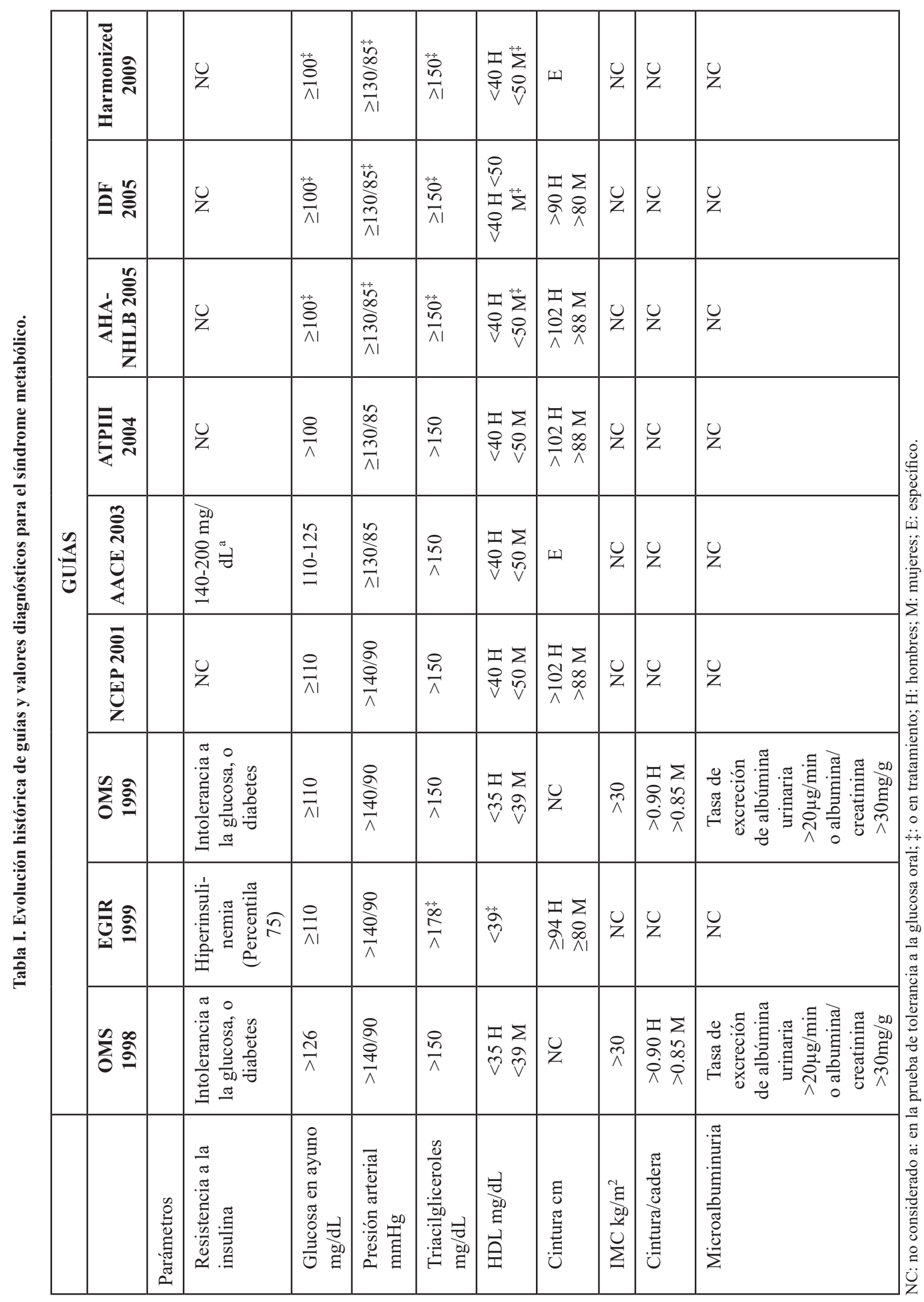


3. Colesterol HDL bajo: $<40 \mathrm{mg} / \mathrm{dL}$ en hombres o $<50 \mathrm{mg} / \mathrm{dL}$ en mujeres.

4. Presión arterial elevada: presión arterial sistólica (PAS) $\geq 130 \mathrm{mmHg}$ y/o presión arterial diastólica (PAD) $\geq 85 \mathrm{mmHg}$

5. Alteración en la homeostasis de la glucosa: glucosa en ayuno $\geq 110 \mathrm{mg} / \mathrm{dL}$

Es importante mencionar que, respecto a los niveles de presión arterial previamente mencionados, se han hecho algunas modificaciones recientes en los criterios de la American Heart Association (AHA). Se considera presión arterial normal, a un valor menor a 120/80 mmHg (Espinosa Brito, 2018). En la Tabla II se detallan categorías y criterios más ampliamente utilizados para la valoración de la presión arterial. De forma similar, a partir de 2019 las nuevas guías de la European Society of Cardiology (ESC) comenzaron a sugerir normas más estrictas para el manejo de los lípidos en todos los niveles de riesgo cardiovascular (Mach et al., 2019), un cambio que se vio pronto reflejado en los puntos de corte más bajos de la dislipidemia en el SM.

\section{Mecanismos de La ReSistencia A La INSULINA Y SU RELACIÓN CON EL SM}

La insulina es uno de los principales reguladores del metabolismo energético, es un inhibidor de la gluconeogénesis hepática, estimula la captura de glucosa, la lipogénesis en el estado postprandial, promueve la glucogénesis e inhibe la lipólisis, entre otros. El desbalance energético observado en estados de sobrepeso y obesidad es acompañado por un estado de hiperinsulinemia. Este estado es precursor de la resistencia a la insulina (Cao, Liu, Hong, \& Liu, 2010) y a su vez para el desarrollo del SM (Johnson \& Olefsky, 2013). Por ejemplo, se ha observado in vitro que la exposición constante a la insulina en los adipocitos disminuye la internalización de la glucosa a través de GLUT4, mientras que la síntesis y el almacenamiento de los lípidos se mantiene sin alteraciones (Gonzalez, Flier, Molle, Accili, \& McGraw, 2011). Esta resistencia a la acción de la insulina puede deberse a alteraciones en la señalización intracelular de la misma en varios niveles. Un ejemplo de ello es el sustrato del receptor a la insulina (IRS). Los cardiomiocitos de la rata neonatal expuestos in vitro a una condición de hipersinsulinemia presentan una disminución en los niveles de las proteínas IRS1 e IRS2; además de una disminución de la fosforilación en uno de los nodos de la señalización de la insulina p38 (Qi et al., 2013). Adicionalmente, el mismo estudio reportó que estas alteraciones en la señalización por insulina estaban asociadas a una menor masa ventricular, apoptosis, fibrosis y falla cardiaca; generando un vínculo entre la resistencia a la insulina y el riesgo cardiovascular.

Debido a los efectos pleitrópicos de la insulina sobre el metabolismo, no es de sorprender que las alteraciones en cualquiera de sus nodos de señalización estén asociadas a múltiples desórdenes metabólicos. Por lo tanto, la resistencia a la insulina es uno de los componentes centrales en el desarrollo del SM. De hecho, dos de los elementos más comunes que presentan los múltiples modelos animales del SM son la hiperinsulinemia y la resistencia a la insulina.

\section{MODELOS EXPERIMENTALES COMO HERRAMIENTA DE INVESTIGACIÓN}

Por lo previamente descrito, se ha vuelto importante explorar mediante modelos experimentales diversas vertientes que permitan un conocimiento más profundo del SM. En particular los mecanismos celulares y moleculares involucrados en las fases tempranas de su desarrollo, con el fin de refinar los criterios diagnósticos y poder establecer alternativas terapéuticas. Los modelos experimentales constituyen una valiosa herramienta para comprender diversos procesos fisiopatológicos asociados al SM. A pesar del desarrollo de estos modelos, es un reto construir criterios unificados, debido a su gran heterogeneidad (Heydemann, 2016; Kleinert et al., 2018; Mašek, Barišić, Micek, \& Starčević, 2020; Small, Brandon, Turner, \& Cooney, 2018).

Existen múltiples modelos animales que se utilizan para el estudio del SM como: primates (Macaca mulatta) (Li et al., 2015), cerdos (Zhang \& Lerman, 2016), conejos (Lozano, Arias-Mutis, Calvo, Chorro, \& Zarzoso, 2019), perros (Kim, Ellmerer, Van Citters, \& Bergman, 2003) e incluso el pez cebra

Tabla II. Criterios del manejo de la presión arterial con respecto al $7^{\circ}$ Reporte del Joint National Committee y las guías del American College of Cardiology/American Heart Association 2017".

\begin{tabular}{|c|c|c|}
\hline PAS y PAD $(\mathbf{m m H g})$ & JNC 7 & ACC/AHA 2017 \\
\hline$<120 \mathrm{y}<80$ & PA normal & PA normal \\
\hline $120-129 \mathrm{y}<80$ & Prehipertensión & PA elevada \\
\hline $130-139$ o $80-89$ & Prehipertensión & HTA Estadío 1 \\
\hline $140-159$ o $90-99$ & HTA Estadío 1 & HTA Estadío 2 \\
\hline$\geq 160$ o $\geq 100$ & HTA Estadío 2 & HTA Estadío 2 \\
\hline
\end{tabular}

Adaptado de: Espinosa Brito, 2018. Hipertensión arterial: cifras para definirla al comenzar. 
(Benchoula et al., 2019). Sin embargo, los más empleados, ya sea por la disponibilidad de reactivos específicos, su facilidad de cuidado y el relativo bajo costo, son los modelos murinos. Dentro de estos últimos, destacan aquellos en los que se induce el SM a través de dietas, de tal manera que emulan factores específicos del estilo de vida que presentan los seres humanos en la patofisiología del SM. Adicionalmente, existen algunos modelos murinos que desarrollan SM, obesidad y diabetes de manera espontánea por alteraciones genéticas en los componentes de la señalización de los sistemas involucrados con la saciedad.Acontinuación, describiremos aspectos generales de los diferentes modelos utilizados para el estudio del desarrollo del SM, con la finalidad de brindar un panorama general del proceso a la comunidad científica.

\section{MODELOS EXPERIMENTALES ALIMENTAdOS CON CARBOHIDRATOS}

Dentro de los modelos animales para inducir el desarrollo de la obesidad y el SM, están las dietas hipercalóricas ricas en carbohidratos o en grasas y las combinadas (como la dieta de cafetería). El objetivo de estos modelos es imitar el desarrollo de las ENT producto de una inadecuada alimentación. Particularmente, estas dietas provocan el desarrollo de la obesidad, hipertensión, alteraciones en la homeostasis de la glucosa, dislipidemia e hígado graso no alcohólico. En el caso de los modelos ricos en carbohidratos, existen múltiples protocolos reportados en la literatura. Se presta particular atención a los basados en monosacáridos, ricos en glucosa (Korntner et al., 2017) o fructosa (Mamikutty et al., 2014), y en los disacáridos como la sacarosa (Velasco et al., 2020).

La selección de un carbohidrato específico otorga el beneficio de permitir discernir las consecuencias de las diferencias metabólicas en el manejo de éstos. Además, existen modelos que combinan alguno de estos regímenes de carbohidratos con un aumento de las calorías provenientes de los lípidos, conocidos como modelos de dieta occidental (Western-diet) o de cafetería (Horne et al., 2020; Ishimoto et al., 2013; Kurita et al., 2019; Moreno-Fernández et al., 2018). Estas dietas combinadas tienen la ventaja de reflejar con mayor fidelidad la complejidad detrás del consumo hipercalórico por los seres humanos, mostrando la sinergia entre estos macronutrimentos. No obstante, esto vuelve más difícil discernir la contribución de cada uno de los componentes individuales en las manifestaciones fenotípicas particulares. Una característica de los modelos ricos en carbohidratos, es la posibilidad de administrar soluciones que contienen azúcares, simulando el consumo de jugos y refrescos por la población humana (Larqué et al., 2011). Cabe resaltar que en 2011, México fue el país con mayor consumo de refresco per capita (Colchero, Popkin, Rivera, \& Ng, 2016). En este sentido, el empleo de un modelo murino del SM basado en el uso de sacarosa en el agua de consumo (Larqué et al., 2011), mimetiza esta tendencia epidemiológica observada en la población mexicana. De esta manera, no solamente se puede elegir un carbohidrato en específico, sino también una forma de consumo. De hecho, los carbohidratos en forma de solución promueven un balance energético positivo; mientras que al administrarse en forma sólida existe la posibilidad de una compensación por la ingesta, manteniendo la ingesta calórica total dentro del rango normal (DiMeglio \& Mattes, 2000). Existen diferentes mecanismos propuestos para explicar este fenómeno, uno de ellos considera el hecho de que al masticar un alimento sólido se desencadena una señal de saciedad, la cual está ausente cuando la sacarosa se presenta de forma líquida (Hollis, 2018). Adicionalmente, se ha reportado que la respuesta secretora del páncreas es mayor en respuesta a un alimento sólido o semi-sólido en comparación con uno líquido (Dhillon, Lee, \& Mattes, 2017).

Una característica notable de las dietas ricas en sacarosa es la capacidad para producir alteraciones hepáticas semejantes al hígado graso no alcohólico, esto debido a que estos modelos se caracterizan por un incremento en la lipogénesis de novo, inducida por el consumo de carbohidratos (Lima et al., 2016; Souza Cruz et al., 2020). Adicionalmente, otro aspecto reciente en los modelos de la dieta rica en sacarosa, es la atención renovada al impacto del dimorfismo sexual en los modelos animales del SM (Mauvais-Jarvis, Arnold, \& Reue, 2017). Esta atención va de la mano de la observación de que la prevalencia del SM varía en los humanos de acuerdo con el sexo y la edad, por lo que resulta necesario comprender ampliamente las diferencias específicas en el desarrollo de la obesidad y el SM, con el fin de diseñar mejores criterios diagnósticos e intervenciones terapéuticas (Pucci et al., 2017). Por ejemplo, en un modelo de ratas Wistar con dieta rica en sacarosa se encontró una mayor intolerancia a la glucosa, dislipidemia y esteatosis hepática en hembras que en machos (Velasco et al., 2020). Este tipo de estudios refuerza la importancia de aumentar los esfuerzos en explorar las diferencias entre los sexos de todos los modelos, que generalmente fueron validados y descritos primero en machos (Clayton \& Collins, 2014). De esta manera, el criterio de elección de un modelo en específico no depende de variables más allá de cuestiones pragmáticas (costo y disponibilidad de la dieta), sino de qué fenómeno en particular se desea estudiar. En la Tabla III se comparan los efectos sobre los parámetros somatométricos y bioquímicos tras un tratamiento con dietas altas en sacarosa y en grasas.

\section{MODELOS EXPERIMENTALES ALIMENTADOS CON DIETA ALTA EN GRASA (DAG)}

Las grasas administradas en la dieta se encuentran en la forma de triacilgliceroles (TAG). Durante el proceso de digestión los TAG se hidrolizan a ácidos grasos libres (AGL) que son el sustrato para la síntesis de lipoproteínas de muy baja densidad (VLDL) hepáticas mediante su reesterificación (lipogénesis). Las partículas de VLDL permiten la circulación de los lípidos en el plasma y su almacenamiento en tejidos periféricos, como el tejido adiposo blanco (Wolfe, Klein, Carraro, \& Weber, 1990). 


\begin{tabular}{|c|c|c|c|c|c|c|c|c|c|c|c|c|}
\hline \multirow{6}{*}{ 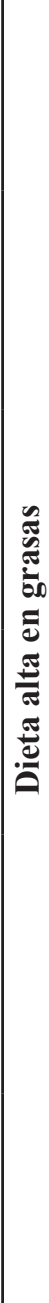 } & \multirow{4}{*}{ 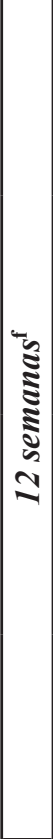 } & 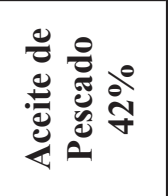 & & 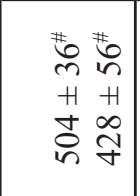 & 至豆 & 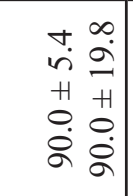 & 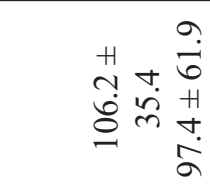 & 之文 & 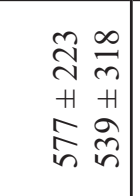 & 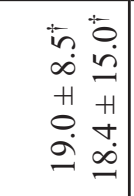 & 安 & 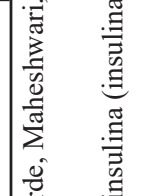 \\
\hline & & 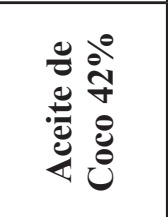 & & 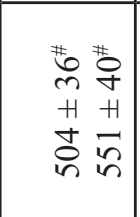 & $\hat{\mathrm{z}} \hat{\mathrm{z}}$ & $\begin{array}{ll}+ & 0 \\
\dot{n} & a \\
H & +1 \\
0 & 0 \\
\dot{0} & 0 \\
\dot{a} & 0\end{array}$ & 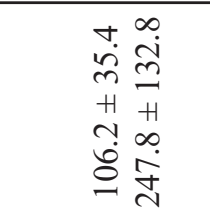 & 文文 & 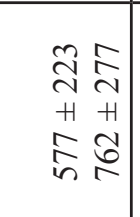 & 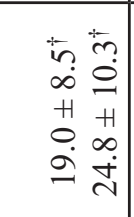 & z安 & 产 \\
\hline & & 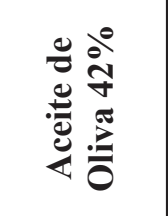 & & 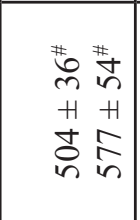 & 立合 & 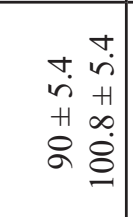 & 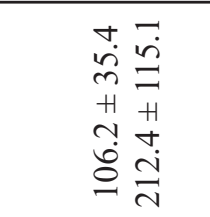 & 文 & 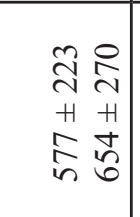 & $\begin{array}{ll}\bar{n} & = \\
\infty & 0 \\
0 & = \\
H & H \\
0 & \infty \\
0 & \infty \\
0 & \dot{v}\end{array}$ & z & $\frac{2}{\hat{a}}$ \\
\hline & & 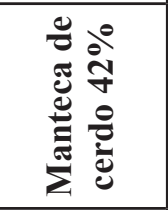 & & 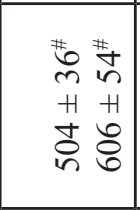 & 至合 & 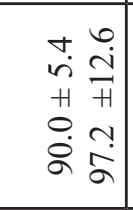 & $\begin{array}{ll}\forall & n \\
i & \infty \\
n & \infty \\
H & H \\
v & a \\
0 & n \\
0 & \infty\end{array}$ & 立 & 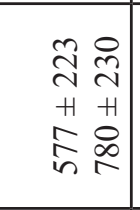 & 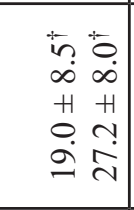 & z & 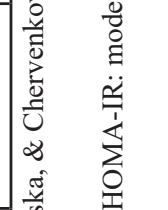 \\
\hline & 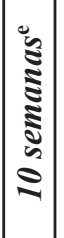 & 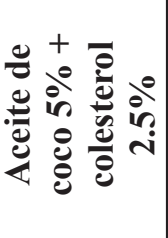 & & 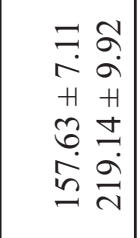 & 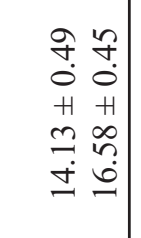 & $\begin{array}{ll}N & \& \\
H & + \\
8 & H \\
O & 0 \\
& m\end{array}$ & 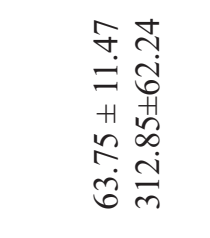 & 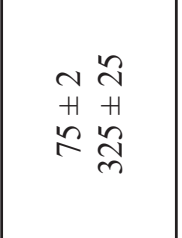 & $\begin{array}{ll}n & 0 \\
+ & 0 \\
H & H \\
0 & 0 \\
0 & 0 \\
\infty & 0 \\
\infty & \vdots\end{array}$ & 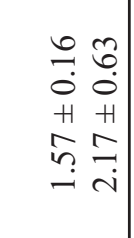 & 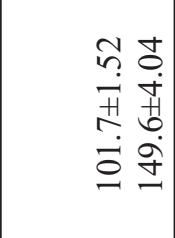 & 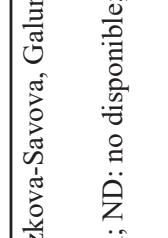 \\
\hline & 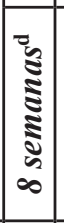 & 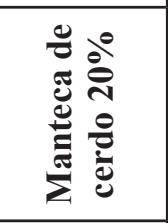 & & 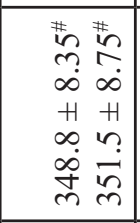 & 至豆 & 穵合 & 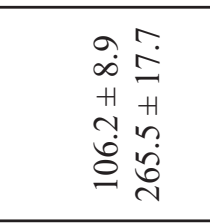 & 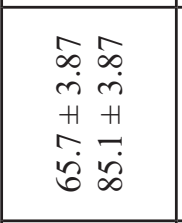 & 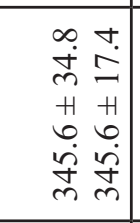 & 至令 & 安 & $\begin{array}{l}0 \\
\bar{z} \\
\frac{\pi}{0}\end{array}$ \\
\hline 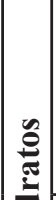 & 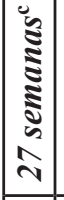 & 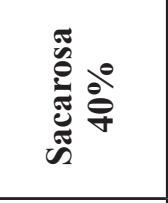 & & 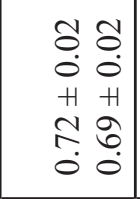 & 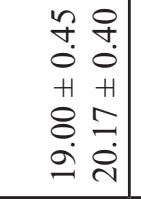 & $\begin{array}{ll}\vec{H} & - \\
H & H \\
\sigma & \infty\end{array}$ & 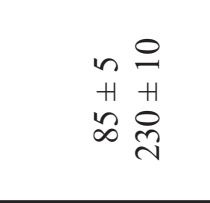 & $\begin{array}{ll}n & n \\
1 & +1 \\
0 & 8\end{array}$ & 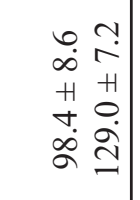 & 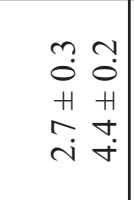 & 安 & 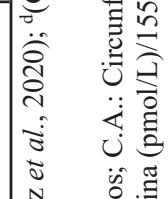 \\
\hline 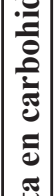 & 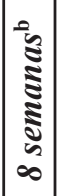 & 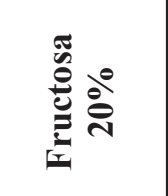 & & 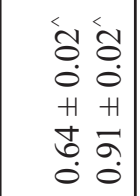 & 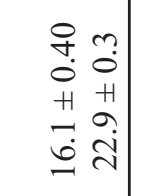 & 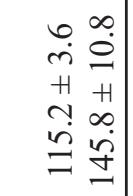 & 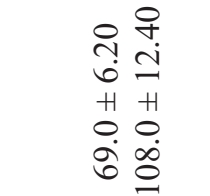 & $\begin{array}{ll}n & 0 \\
n & \infty \\
-1 & \dot{n} \\
+1 & +1 \\
0 & 0 \\
\infty & \infty \\
n & \infty\end{array}$ & 号 & 学 & 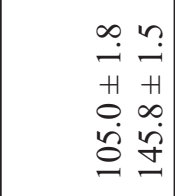 & 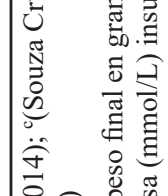 \\
\hline 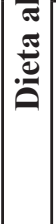 & 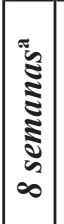 & 营 & & 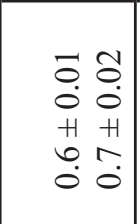 & $\begin{array}{ll}+ & + \\
0 & 0 \\
H & + \\
0 & 0 \\
0 & 0 \\
0 & \dot{v}\end{array}$ & 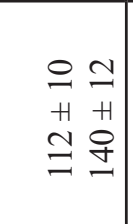 & $\begin{array}{l}\simeq \stackrel{1}{ } \\
\text { H } \\
\infty \\
\infty \\
\infty\end{array}$ & $\begin{array}{l}n \\
+1 \\
i n \\
\text { in }\end{array}$ & 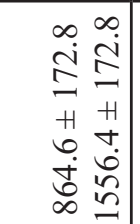 & 至 & 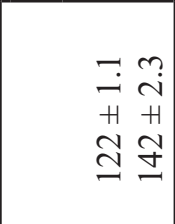 & $\dot{\vec{v}}$ \\
\hline & & & 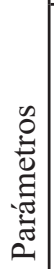 & 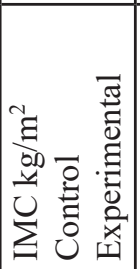 & 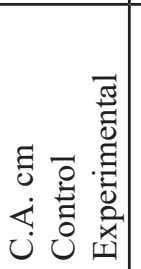 & 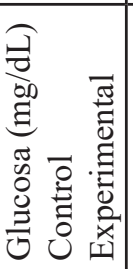 & 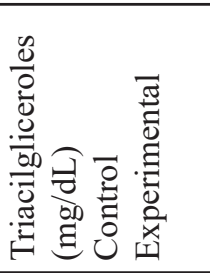 & 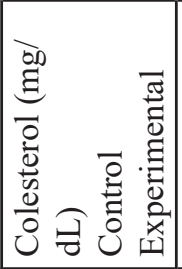 & 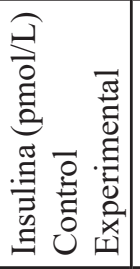 & 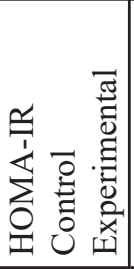 & 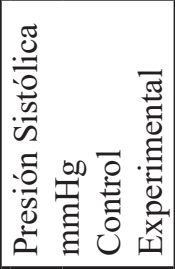 & 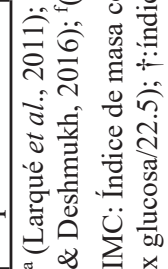 \\
\hline
\end{tabular}


Cabe resaltar que las DAG, al igual que las dietas basadas en carbohidratos son un modelo heterogéneo. Es decir, existen múltiples variantes en cuanto al tipo de ácidos grasos que las constituyen (\% de saturados, mono- o poli-insaturados), así como en cuanto al porcentaje de calorías aportadas por los mismos. Por ejemplo, existen modelos de DAG donde entre el $40-60 \%$ de las calorías totales provienen de grasas. Asimismo, el origen de las grasas varía desde las de origen animal (manteca de cerdo, aceite de pescado y cebo) a los aceites de origen vegetal (coco, oliva, cártamo). Es importante mencionar que los porcentajes de calorías proporcionados por las grasas son hasta cierto punto arbitrarios. Históricamente, uno de los pioneros en el diseño y manufactura de dietas altas en grasas fue la compañía Research Diets ${ }^{\circledR}$. Esta compañía hace 20 años lanzó una línea de dietas con un 10, 45 y $60 \%$ de las calorías proporcionadas por grasas (Speakman, 2019). Estas dietas rápidamente se volvieron un punto de referencia para los estudios de obesidad, SM y DM2. Sin embargo, múltiples grupos de investigación han diseñado sus propias dietas altas en grasa para responder a preguntas específicas de investigación. Esta heterogeneidad de composiciones ha ocasionado una cierta inconsistencia de los efectos de las DAG en modelos animales. En 2006, Buettner y cols., compararon los efectos que tenían múltiples DAG (con $42 \%$ de las calorías totales provenientes de grasa) sobre el metabolismo en ratas Wistar (Tabla III). Se compararon 4 dietas con diferentes fuentes de grasas (manteca de cerdo, aceite de oliva, aceite de coco y aceite de pescado) con una dieta estándar (con 11\% de las calorías provenientes de grasas). Mientras que las 4 DAG presentaron un consumo calórico similar, al finalizar las 12 semanas de tratamiento hubo diferencias en el peso de los animales. Aquellos alimentados con manteca de cerdo, aceite de coco y de oliva presentaron un peso significativamente mayor al del grupo control; mientras que el grupo alimentado con aceite de pescado tuvo un peso menor. Por otro lado, los grupos alimentados con manteca y aceite de olivo presentaron una curva de sensibilidad a la insulina alterada. Finalmente, únicamente los grupos alimentados con manteca, aceite de coco y de oliva presentaron grados variables de esteatosis hepática (Buettner et al., 2006). Estos resultados indican que no necesariamente por el simple hecho de tener una mayor densidad calórica, una DAG dará lugar a todos los signos asociados al SM. Las grasas saturadas y monoinsaturadas tienden a provocar obesidad y desórdenes metabólicos a diferencia de las grasas poliinsaturadas que tienen en general un efecto protector. Este efecto diferencial puede deberse en parte a cambios en el metabolismo de los adipocitos en respuesta a los diferentes tipos de grasas. Por ejemplo, la línea celular de adipocitos 3T3-L1 presenta una mayor secreción de TNF $\alpha$ tras la exposición a aceite de coco en comparación con el aceite de oliva (García-Escobar et al., 2017). Adicionalmente, el consumo de grasas insaturadas (aceite de oliva) promueve una mayor termogénesis en el tejido adiposo marrón en comparación con las grasas saturadas (Shin \& Ajuwon, 2018). Finalmente, el consumo de ácidos grasos omega-3 previene la hipertrofia de los adipocitos (Balogun \& Cheema, 2016).
Así como los resultados son variables de acuerdo con el tipo de grasa utilizada, existe discordancia entre los resultados de modelos DAG dependiendo de la duración del tratamiento, con regímenes de administración que van de 4 a 40 semanas y un porcentaje de calorías del 37\% al 60\% (Wong, Chin, Suhaimi, Fairus, \& Ima-Nirwana, 2016). Una característica notable de las DAG es el hecho de que en múltiples instancias el consumo de alimento se reduce para compensar el aumento de la densidad calórica. Sin embargo, a pesar de esta compensación, las DAG son capaces de generar una ganancia de peso, que se puede explicar mediante múltiples mecanismos. Por ejemplo a nivel hepático, una DAG basada en grasas saturadas inhibe la fosforilación de la enzima acetil-CoA carboxilasa, de esta manera se promueve la biosíntesis de ácidos grasos (Guo et al., 2009). La oxidación de ácidos grasos de cadena larga requiere de la proteína carnitina palmitoiltransferasa I (CPT-1) para su transporte hacia la matriz mitocondrial, se ha observado que la DAG disminuye la expresión del gen que codifica para esta enzima (Yang et al., 2019). Otro mecanismo mediante el cual las DAG son capaces de alterar el metabolismo es el fenómeno de lipotoxicidad; es decir, por acumulación de lípidos en tejidos ectópicos (músculo estriado, hígado, páncreas, etc.), ocasionado por sobrepasar la capacidad del tejido adiposo de almacenar TAG (Engin, 2017). En este aspecto, se observó que las ratas alimentadas con una dieta DAG durante 20 semanas, desarrollaron depósitos de lípidos no sólo en el hígado sino también en el páncreas. Esta acumulación estuvo asociada a un incremento del tejido fibroso alrededor de los islotes y a una homeostasis alterada de la glucosa (Zhou et al., 2019).

Por otro lado, un estudio encontró que después de finalizar un periodo de 4 semanas de consumo de DAG (con $60 \%$ de las calorías provenientes de los lípidos), el peso corporal de los animales no mostraba diferencias significativas entre los grupos. Sin embargo, las ratas con acceso a la dieta rica en grasa acumularon significativamente más tejido adiposo en las regiones gonadal, retroabdominal y visceral (Díaz-Urbina et al., 2018; Hu et al., 2018). Adicionalmente, entre los desórdenes metabólicos inducidos por DAG, se ha observado un incremento en la concentración de leptina en el plasma, como consecuencia del aumento en la proporción de grasa corporal (Stemmer et al., 2012).

Por otro lado, las DAG han sido utilizadas en estudios no solamente para desarrollar SM, sino también para determinar los efectos metabólicos multigeneracionales sobre la progenie. Ratas hembra alimentadas con DAG (32\% de las calorías provenientes de lípidos) durante 90 días (incluyendo los periodos de apareamiento, gestación y lactancia), además de presentar una mayor ganancia de peso y alteraciones metabólicas. Alos 90 días de edad, su progenie presentó un mayor peso y adiposidad comparado con el de animales gestados por hembras del grupo control. Asimismo, la progenie de las hembras alimentadas con 
DAG presentó hiperleptinemia y dislipidemias, entre los 9-14 días postnatales (Kirk et al., 2009).

Las DAG tienen el potencial de mimetizar varios de los desórdenes asociados al SM. Sin embargo, existen múltiples variantes de esta dieta, por lo que en la elección de una DAG debe de considerarse además del aporte calórico total, el origen de las grasas, régimen de alimentación ( ad libitum o controlada) y la duración del tratamiento. En este sentido, se ha observado que ratas Sprague-Dawley alimentadas con DAG bajo dos regímenes, ad libitum y en pulsos de 2 horas al día (durante la fase nocturna), presentan un comportamiento diferente. Las ratas con acceso libre al alimento en promedio tienen un mayor consumo calórico, una mayor ganancia de peso, así como una mayor proporción de tejido adiposo (Bake, Morgan, \& Mercer, 2014). Además, no sólo es importante el periodo de acceso al alimento, sino el momento del día en el que se realiza. En ratones (animales nocturnos), se ha observado que el acceso al alimento DAG en la fase de luz genera una mayor ganancia de peso y resistencia a la insulina, que aquellos con acceso al alimento en la fase nocturna (Arble, Bass, Laposky, Vitaterna, \& Turek, 2009; Yoon et al., 2012).

\section{Modelos GeNÉTICOS}

$\mathrm{Si}$ bien entre los principales factores involucrados en el desarrollo del SM se encuentran aspectos de estilo de vida, el fondo genético del individuo debe ser considerado. Existen ejemplos de desórdenes congénitos asociados a la obesidad y al SM, como por ejemplo el síndrome de Prader-Willi (Crinò, Fintini, Bocchini, \& Grugni, 2018) y el síndrome de Alström (Minton et al., 2006). Además, se han identificado a la fecha múltiples polimorfismos asociados al desarrollo de la resistencia a la insulina y al SM (Brown \& Walker, 2016).

De esta manera, diversos modelos animales nos han proporcionado información sobre los mecanismos genéticos asociados al desarrollo de la obesidad y al SM. Uno de los primeros modelos desarrollados fueron los ratones $(\mathrm{ob} / \mathrm{ob})$, con una mutación en el gen que codifica para la leptina. Al carecer de la señal de saciedad mediada por esta proteína, los ratones desarrollan hiperfagia, obesidad, hipogonadismo y diversas anomalías metabólicas (Ahima \& Flier, 2000). A este modelo siguió el de los ratones $(d b / d b)$, que carecían de un receptor funcional para la leptina, y con un fenotipo similar a los ratones $(o b / o b)$ pero que además desarrollaron DM2 de forma espontánea (Kurtz, Morris, \& Pershadsingh, 1989). De igual manera, en las ratas obesas de la cepa designada Zucker no sólo se presentó obesidad por hiperfagia, sino también una deficiencia en el gen que codifica para el receptor de leptina (Dong et al., 2010).

A estos primeros modelos, siguieron otros que representaban mejor las alteraciones del SM. Como es el caso de las ratas DahlS.Z-Lepr ${ }^{f a} /$ Lepr $^{f a}$, las cuales surgieron de la cruza de ratas Zucker obesas con ratas de la cepa Dahl (sensibles a la sal). Además de un mayor peso y adiposidad, estas ratas desarrollaron resistencia a la insulina, hiperinsulinemia, dislipidemias, hipertensión y esteatosis hepática (Hattori et al. 2011). A este modelo siguió el de las ratas Goto-Kakizaki, generadas por la cruza selectiva de ratas que presentaban intolerancia a la glucosa (Akash, Rehman, \& Chen, 2013). Estas ratas presentaron un deterioro progresivo de la capacidad secretora de las células beta pancreáticas que las llevó a una hiperglucemia y al desarrollo de hiperleptinemia e hiperfagia (Maekawa et al., 2006). Finalmente, la compañía Charles River aisló ratones con una mutación en el gen que codifica para el receptor a leptina, el Pound Mice ${ }^{\mathrm{TM}}$ (C57BL/6NCrlLepr $\left.^{\mathrm{db}-\mathrm{lb}}\right)$. Estos ratones presentaron varias características del SM: obesidad, hiperinsulinemia, hiperglucemia, hiperleptinemia y colesterol total elevado (THE POUND MOUSE $\mid$ Charles River Laboratories., s/f). Las mutaciones en los genes que codifican para leptina y su receptor son raros en humanos, por lo que no necesariamente representan la patogénesis del SM observada en la gran mayoría de los casos.

Como mencionamos previamente, la etiología, la complejidad e investigación del SM tanto en humanos como en modelos animales ofrece diversas líneas interesantes de abordar. Como se describió en secciones anteriores, existen múltiples modelos que desarrollan un fenotipo del SM. Cada uno de ellos emula un aspecto de la fisiopatología del SM, como el consumo de bebidas azucaradas, la ingesta de dietas hipercalóricas e incluso algunas alteraciones genéticas. Sin embargo, no existe un criterio único en cuanto a los modelos animales para el estudio del SM. En la literatura se encuentran dietas con diversas composiciones de macronutrientes, así como regímenes de alimentación. Debido a lo anterior la elección de un modelo en específico requiere considerar qué aspecto del SM se desea estudiar y la metodología, e incluso los costos que cada uno conlleva.

Nuestro grupo de trabajo se enfoca al estudio del SM utilizando modelos experimentales empleando una dieta alta en grasas (Buettner et al., 2006) y otra alta en sacarosa (Larqué et al., 2011). El resultado del uso de ambas dietas es un incremento del peso corporal respecto al grupo control al finalizar los dos meses de tratamiento. En ambos modelos, el aumento de peso ocasionado por la dieta incrementa el tamaño de los depósitos de grasa viscerales. Además, induce respuestas fisiológicas alteradas incluyendo intolerancia a la glucosa, hiperinsulinemia e hiperleptinemia. El modelo rico en sacarosa tiende a generar desórdenes en el metabolismo de la glucosa más rápidamente; mientras que el modelo rico en grasas genera una mayor acumulación de tejido adiposo visceral (datos sin publicar).

\section{Conclusiones}

El empleo de modelos animales para el estudio del SM permite la evaluación de los cambios que ocurren a nivel histológico, fisiológico y bioquímico durante diferentes etapas del desarrollo 
de la enfermedad. Aunque existen varios de ellos y todos aportan conocimiento, es importante la elección del que se adapte a las líneas de investigación del SM y sea de interés o que impacte sobre las comorbilidades asociadas al SM de mayor importancia en la actualidad.

\section{Agradecimientos}

Proyecto DGAPA PAPIIT IA207321, IA205321.

\section{REFERENCIAS}

Ahima, R. S. \& Flier, J. S. (2000). Leptin. Annual Review of Physiology, 62, 413-437. DOI: 10.1146/annurev. physiol.62.1.413

Akash, M. S., Rehman, K. \& Chen, S. (2013). GotoKakizaki rats: Its suitability as non-obese diabetic animal model for spontaneous type 2 diabetes mellitus. Current Diabetes Reviews, 9(5), 387-396. DOI: $10.2174 / 15733998113099990069$

Alberti, K. G. \& Zimmet, P. Z. (1998). Definition, diagnosis and classification of diabetes mellitus and its complications. Part 1: Diagnosis and classification of diabetes mellitus provisional report of a WHO consultation. Diabetic Medicine: A Journal of the British Diabetic Association, 15(7), 539-553. DOI: 10.1002/(SICI)10969136(199807)15:7<539::AID-DIA668>3.0.CO;2-S

Alberti, K. G. M. M., Zimmet, P. \& Shaw, J. (2006). Metabolic syndrome-a new world-wide definition. A Consensus Statement from the International Diabetes Federation. Diabetic Medicine, 23(5), 469-480. DOI: 10.1111/j.14645491.2006.01858.x

Arble, D. M., Bass, J., Laposky, A. D., Vitaterna, M. H. \& Turek, F. W. (2009). Circadian timing of food intake contributes to weight gain. Obesity (Silver Spring, Md.), 17(11), 2100-2102. DOI: 10.1038/oby.2009.264

Aronis, K. N. \& Mantzoros, C. S. (2012).Abriefhistory of insulin resistance: From the first insulin radioimmunoassay to selectively targeting protein kinase C pathways. Metabolism, 61(4), 445-449. DOI: 10.1016/j.metabol.2012.01.001

Bake, T., Morgan, D. G. A. \& Mercer, J. G. (2014). Feeding and metabolic consequences of scheduled consumption of large, binge-type meals of high fat diet in the Sprague-Dawley rat. Physiology \& Behavior, 128, 70-79. DOI: 10.1016/j. physbeh.2014.01.018

Balogun, K. A. \& Cheema, S. K. (2016). Dietary Omega-3 Fatty Acids PreventedAdipocyte Hypertrophyby Downregulating DGAT-2 and FABP-4 in a Sex-Dependent Fashion. Lipids, 51(1), 25-38. DOI: 10.1007/s11745-015-4105-x

Benchoula, K., Khatib, A., Jaffar, A., Ahmed, Q. U., Sulaiman, W. M. A. W., Wahab, R. A. \& El-Seedi, H. R. (2019). The promise of zebrafish as a model of metabolic syndrome. Experimental Animals, 68(4), 407-416. DOI: 10.1538/ expanim.18-0168

Booth, J. (1977). A short history of blood pressure measurement. Proceedings of the Royal Society of Medicine, 70(11),
793-799. Recuperado de https://www.ncbi.nlm.nih.gov/ pmc/articles/PMC1543468/

Brown,A.E.\& Walker, M.(2016). Genetics of Insulin Resistance and the Metabolic Syndrome. Current Cardiology Reports, 18(8), 75. DOI: 10.1007/s11886-016-0755-4

Buettner, R., Parhofer, K. G., Woenckhaus, M., Wrede, C. E., Kunz-Schughart, L. A., Schölmerich, J. \& Bollheimer, L. C. (2006). Defining high-fat-diet rat models: Metabolic and molecular effects of different fat types. Journal of Molecular Endocrinology, 36(3), 485-501. DOI: 10.1677/ jme.1.01909

Cao, W., Liu, H.-Y., Hong, T. \& Liu, Z. (2010). Excess exposure to insulin may be the primary cause of insulin resistance. American Journal of Physiology. Endocrinology and Metabolism, 298(2), E372. DOI: 10.1152/ajpendo.00677.2009

Clayton, J. A. \& Collins, F. S. (2014). Policy: NIH to balance sex in cell and animal studies. Nature, 509(7500), 282-283. DOI: $10.1038 / 509282 \mathrm{a}$

Colchero, M. A., Popkin, B. M., Rivera, J. A. \& Ng, S. W. (2016). Beverage purchases from stores in Mexico under the excise tax on sugar sweetened beverages: Observational study. BMJ (Clinical research ed.), 352, h6704. DOI: 10.1136/bmj.h6704

Crinò, A., Fintini, D., Bocchini, S. \& Grugni, G. (2018). Obesity management in Prader-Willi syndrome: Current perspectives. Diabetes, Metabolic Syndrome and Obesity: Targets and Therapy, 11, 579-593. DOI: 10.2147/DMSO. S141352

Dawber, T. R., Moore, F. E. \& Mann, G. V. (1957). Coronary heart disease in the Framingham study. American Journal of Public Health and the Nation's Health, 47(4 Pt 2), 4-24. DOI: 10.2105 /ajph.47.4_pt_2.4

Dhillon, J., Lee, J. Y. \& Mattes, R. D. (2017). The cephalic phase insulin response to nutritive and low-calorie sweeteners in solid and beverage form. Physiology \& Behavior, 181, 100-109. DOI: 10.1016/j.physbeh.2017.09.009

Díaz-Urbina, D., Escartín-Pérez, R. E., López-Alonso, V. E. \& Mancilla-Díaz, J. M. (2018). Efectos de una dieta con alto contenido de grasas sobre patrones conductuales alimentarios. Acta Colombiana de Psicología, 21(1), 95-115. DOI: 10.14718/ACP.2018.21.1.5

DiMeglio, D. P. \& Mattes, R. D. (2000). Liquid versus solid carbohydrate: Effects on food intake and body weight. International Journal of Obesity and Related Metabolic Disorders: Journal of the International Association for the Study of Obesity, 24(6), 794-800. DOI: 10.1038/ sj.ijo.0801229

Dong, Y. F., Liu, L., Kataoka, K., Nakamura, T., Fukuda, M., Tokutomi, Y., Nako, H., Ogawa, H. \& Kim-Mitsuyama, S. (2010). Aliskiren prevents cardiovascular complications and pancreatic injury in a mouse model of obesity and type 2 diabetes. Diabetologia, 53(1), 180-191. DOI: 10.1007/ s00125-009-1575-5 
Eknoyan, G. (2007). Adolphe Quetelet (1796 1874) the average man and indices of obesity. Nephrology Dialysis Transplantation, 23(1), 47-51. DOI: 10.1093/ndt/gfm517

Engin, A. B. (2017). What Is Lipotoxicity? En A. B. Engin \& A. Engin(Eds.), Obesity andLipotoxicity (pp. 197-220). Cham: Springer International Publishing. DOI: 10.1007/978-3319-48382-5_8

Enzi, G., Busetto, L., Inelmen, E. M., Coin, A. \& Sergi, G. (2003). Historical perspective: Visceral obesity and related comorbidity in Joannes Baptista Morgagni's "De sedibus et causis morborum per anatomen indagata". International Journal of Obesity and Related Metabolic Disorders: Journal of the International Association for the Study of Obesity, 27(4), 534-535. DOI: 10.1038/sj.ijo.0802268

Espinosa Brito, A. (2018). Hipertensión arterial: Cifras para definirla al comenzar 2018. Revista Finlay, 8(1), 66-74. Recuperado de http://scielo.sld.cu/scielo.php?script=sci abstract\&pid=S2221-24342018000100008\&lng=es\&nr $\mathrm{m}=$ iso\&tlng=es

Gancheva, S., Zhelyazkova-Savova, M., Galunska, B. \& Chervenkov, T. (2015). Experimental models of metabolic syndrome in rats. Scripta Scientifica Medica, 47(2), 14-21. DOI: $10.14748 /$ ssm.v47i2.1145

García-Escobar, E., Monastero, R., García-Serrano, S., GómezZumaquero, J. M., Lago-Sampedro, A., Rubio-Martín, E., Colomo, N., Rodríguez-Pacheco, F., Soriguer, F. \& RojoMartínez, G. (2017). Dietary fatty acids modulate adipocyte TNFa production via regulation of its DNA promoter methylation levels. The Journal of Nutritional Biochemistry, 47, 106-112. DOI: 10.1016/j.jnutbio.2017.05.006

Gonzalez, E., Flier, E., Molle, D., Accili, D. \& McGraw, T. E. (2011). Hyperinsulinemia leads to uncoupled insulin regulation of the GLUT4 glucose transporter and the FoxO1 transcription factor. Proceedings of the National Academy of Sciences of the United States of America, 108(25), 10162-10167. DOI: 10.1073/pnas.1019268108

Guo, H., Liu, D., Ma, Y., Liu, J., Wang, Y., Du, Z., Wang, X., Shen, J. \& Peng, H. (2009). Long-term baicalin administration ameliorates metabolic disorders and hepatic steatosis in rats given a high-fat diet. Acta Pharmacologica Sinica, 30(11), 1505-1512. DOI: 10.1038/aps.2009.150

Haller, H. (1977). [Epidermiology and associated risk factors of hyperlipoproteinemia]. Zeitschrift fur die gesamte innere Medizin und ihre Grenzgebiete, 32(8), 124-128.

Hattori, T., Murase, T., Ohtake, M., Inoue, T., Tsukamoto, H., Takatsu, M., Kato, Y., Hashimoto, K., Murohara, T. \& Nagata, K. (2011). Characterization of a new animal model of metabolic syndrome: The DahlS.Z-Lepr(fa)/ Lepr(fa) rat. Nutrition \& Diabetes, 1, e1. DOI: 10.1038/ nutd.2010.1

Heydemann, A. (2016). An Overview of Murine High Fat Diet as a Model for Type 2 Diabetes Mellitus. Journal of Diabetes Research, 2016, 2902351. DOI: 10.1155/2016/2902351

Hollis, J. H. (2018). The effect of mastication on food intake, satiety and body weight. Physiology \& Behavior, 193(Pt B), 242-245. DOI: 10.1016/j.physbeh.2018.04.027

Horne, R. G., Yu, Y., Zhang, R., Abdalqadir, N., Rossi, L., Surette, M., Sherman, P. M. \& Adeli, K. (2020). High FatHigh Fructose Diet-Induced Changes in the Gut Microbiota Associated with Dyslipidemia in Syrian Hamsters. Nutrients, 12(11). DOI: 10.3390/nu12113557

Hu, S., Wang, L., Yang, D., Li, L., Togo, J., Wu, Y., Liu, Q., Li, B., Li, M., Wang, G., Zhang, X., Niu, C., Li, J., Xu, Y., Couper, E., Whittington-Davies, A., Mazadi, M., Luo, L., Whang, S., Douglas, A. \& Speakman, JR. (2018). Dietary Fat, but Not Protein or Carbohydrate, Regulates Energy Intake and Causes Adiposity in Mice. Cell metabolism, 28(3), 415-431.e4. DOI: 10.1016/j.cmet.2018.06.010

Ishimoto, T., Lanaspa, M. A., Rivard, C. J., Roncal-Jimenez, C. A., Orlicky, D. J., Cicerchi, C., McMahan, R H., Abdelmalek, M. F., Rosen, H. R., Jackman, M. R., MacLean, P. S., Diggle, C. P., Asipu, A., Inaba S., Kosugi, T., Sato, W., Marumaya, S., Sánchez-Lozada, L. G., Sautin, Y. Y., Hill, J. O., Bonthron, D. T. \& Johnson, R. J. (2013). High-fat and high-sucrose (western) diet induces steatohepatitis that is dependent on fructokinase. Hepatology (Baltimore, Md.), 58(5), 1632-1643. DOI: 10.1002/hep.26594

Johnson, A. M. F. \& Olefsky, J. M. (2013). The origins and drivers of insulin resistance. Cell, 152(4), 673-684. DOI: 10.1016/j.cell.2013.01.041

Kim, S. P., Ellmerer, M., Van Citters, G. W. \& Bergman, R. N. (2003). Primacy of hepatic insulin resistance in the development of the metabolic syndrome induced by an isocaloric moderate-fat diet in the dog. Diabetes, 52(10), 2453-2460. DOI: 10.2337/diabetes.52.10.2453

Kirk, S. L., Samuelsson, A.-M., Argenton, M., Dhonye, H., Kalamatianos, T., Poston, L., Taylor, P. D. \& Coen, C. W. (2009). Maternal obesity induced by diet in rats permanently influences central processes regulating food intake in offspring. PloS One, 4(6), e5870. DOI: 10.1371/journal. pone. 0005870

Kleinert, M., Clemmensen, C., Hofmann, S. M., Moore, M. C., Renner, S., Woods, S. C., Huypens, P., Beckers, J., de Angelis, M. H., Schürmann, A., Bakhti, M., Klingenspor, M., Heiman, M., Cherrington, A. D., Ristow, M., Lickert, H., Wolf, E., Havel, P. J., Müller, T. D. \& Tschöp, M. H. (2018). Animal models of obesity and diabetes mellitus. Nature Reviews. Endocrinology, 14(3), 140-162. DOI: 10.1038/nrendo.2017.161

Korntner, S., Kunkel, N., Lehner, C., Gehwolf, R., Wagner, A., Augat, P., Stephan, D., Heu, V., Bauer, H.-C., Traweger, A. \& Tempfer, H. (2017). A high-glucose diet affects Achilles tendon healing in rats. Scientific Reports, 7(1), 780. DOI: 10.1038/s41598-017-00700-z

Kurita, Y., Ohki, T., Soejima, E., Yuan, X., Kakino, S., Wada, N., Hashinaga, T., Nakayama, H., Tani, J., Tajiri, Y., Hiromatsu, Y., Yamada, K. \& Nomura, M. (2019). A HighFat/High-Sucrose Diet Induces WNT4 Expression in Mouse 
Pancreatic $\beta$-cells. The Kurume Medical Journal, 65(2), 55-62. DOI: 10.2739/kurumemedj.MS652008

Kurtz, T. W., Morris, R. C. \& Pershadsingh, H. A. (1989). The Zucker fatty rat as a genetic model of obesity and hypertension. Hypertension (Dallas, Tex.: 1979), 13(6 Pt 2), 896-901. DOI: 10.1161/01.hyp.13.6.896

Larqué, C., Velasco, M., Navarro-Tableros, V., Duhne, M., Aguirre, J., Gutiérrez-Reyes, G., Moreno, J., Robles-Diaz, G., Hong, E. \& Hiriart, M. (2011). Early endocrine and molecular changes in metabolic syndrome models. IUBMB Life, 63(10), 831-839. DOI: 10.1002/iub.544

Li, L., Liao, G., Yang, G., Lu, Y., Du, X., Liu, J., Li, L., Wang, C., Li, L., Ren, Y., Zhong, Z., Cheng, J. \& Chen, Y. (2015). Highfat diet combined with low-dose streptozotocin injections induces metabolic syndrome in Macaca mulatta. Endocrine, 49(3), 659-668. DOI: 10.1007/s12020-015-0542-9

Lima, M. L. R. P., Leite, L. H. R., Gioda, C. R., Leme, F. O. P., Couto, C. A., Coimbra, C. C., Leite, V. H. R. \& Ferrari, T. C.A. (2016).ANovel Wistar Rat Model of Obesity-Related Nonalcoholic Fatty Liver Disease Induced by Sucrose-Rich Diet. Journal of Diabetes Research, 2016, 9127076. DOI: 10.1155/2016/9127076

Lozano, W. M., Arias-Mutis, O. J., Calvo, C. J., Chorro, F. J. \& Zarzoso, M. (2019). Diet-Induced Rabbit Models for the Study of Metabolic Syndrome. Animals: An Open Access Journal from MDPI, 9(7), E463. DOI: 10.3390/ani9070463

Mach, F., Baigent, C., Catapano, A. L., Koskinas, K. C., Casula, M., Badimon, L., Chapman, M. J., De Backer, G. G., Delgado, V., Ference, B. A., Graham, I. M., Halliday, A., Landmesser, U., Mihaylova, B., Pedersen, T. R., Riccardi, G., Richter, D. J., Sabatine, M. S., Taskinen, M.R., Tokgozoglu, L. \& Wiklund, O. (2020). ESC Scientific Document Group (2020). 2019 ESC/EAS Guidelines for the management of dyslipidaemias: Lipid modification to reduce cardiovascular risk. European Heart Journal, 41(1), 111-118. DOI: 10.1093/eurheartj/ehz455

Maekawa, F., Fujiwara, K., Kohno, D., Kuramochi, M., Kurita, H. \& Yada, T. (2006). Young adult-specific hyperphagia in diabetic Goto-kakizaki rats is associated with leptin resistance and elevation of neuropeptide Y mRNA in the arcuate nucleus. Journal of Neuroendocrinology, 18(10), 748-756. DOI: 10.1111/j.1365-2826.2006.01470.x

Mamikutty, N., Thent, Z. C., Sapri, S. R., Sahruddin, N. N., Mohd Yusof, M. R. \& Haji Suhaimi, F. (2014). The establishment of metabolic syndrome model by induction of fructose drinking water in male Wistar rats. BioMed Research International, 2014, 263897. DOI: 10.1155/2014/263897

Mašek, T., Barišić, J., Micek, V. \& Starčević, K. (2020). Cafeteria Diet and High-Fructose Rodent Models of NAFLD Differ in the Metabolism of Important PUFAand Palmitoleic Acid without Additional Influence of Sex. Nutrients, 12(11), E3339. DOI: $10.3390 /$ nu12113339

Mauvais-Jarvis, F., Arnold, A. P. \& Reue, K. (2017). A Guide for the Design of Pre-clinical Studies on Sex Differences in Metabolism. Cell Metabolism, 25(6), 1216-1230. DOI: 10.1016/j.cmet.2017.04.033

Minton, J. a. L., Owen, K. R., Ricketts, C. J., Crabtree, N., Shaikh, G., Ehtisham, S., Porter, J. R., Carey, C., Hodge, D., Paisey, R., Walker, M. \& Barrett, T. G. (2006). Syndromic obesity and diabetes: Changes in body composition with age and mutation analysis of ALMS1 in 12 United Kingdom kindreds with Alstrom syndrome. The Journal of Clinical Endocrinology and Metabolism, 91(8), 3110-3116. DOI: 10.1210/jc.2005-2633

Moreno-Fernández, S., Garcés-Rimón, M., Vera, G., Astier, J., Landrier, J. F. \& Miguel, M. (2018). High Fat/High Glucose Diet Induces Metabolic Syndrome in an Experimental Rat Model. Nutrients, 10(10). DOI: 10.3390/nu10101502

O’Neill, S., Bohl, M., Gregersen, S., Hermansen, K. \& O’Driscoll, L. (2016). Blood-Based Biomarkers for Metabolic Syndrome. Trends in Endocrinology \& Metabolism, 27(6), 363-374. DOI: 10.1016/J.TEM.2016.03.012

Pucci, G., Alcidi, R., Tap, L., Battista, F., Mattace-Raso, F. \& Schillaci, G. (2017). Sex- and gender-related prevalence, cardiovascular risk and therapeutic approach in metabolic syndrome: A review of the literature. Pharmacological Research, 120, 34-42. DOI: 10.1016/j.phrs.2017.03.008

Qi, Y., Xu, Z., Zhu, Q., Thomas, C., Kumar, R., Feng, H., Dostal, D. E., White, M. F., Baker, K. M. \& Guo, S. (2013). Myocardial loss of IRS1 and IRS2 causes heart failure and is controlled by $\mathrm{p} 38 \alpha$ MAPK during insulin resistance. Diabetes, 62(11), 3887-3900. DOI: 10.2337/db13-0095

Reaven, G. M. (1988). Banting lecture 1988. Role of insulin resistance in human disease. Diabetes, 37(12), 1595-1607. DOI: $10.2337 /$ diab.37.12.1595

Rössner, S. (2009). Eskil Kylin (1889-1975). Obesity Reviews, 10(3), 362-362. doi: 10.1111/j.1467-789X.2008.00531.x

Saklayen, M. G. (2018). The Global Epidemic of the Metabolic Syndrome. Current Hypertension Reports, 20(2), 12. DOI: 10.1007/s11906-018-0812-z

Schaefer, E. J., Tsunoda, F., Diffenderfer, M., Polisecki, E., Thai, N. \& Asztalos, B. (2000). The Measurement of Lipids, Lipoproteins, Apolipoproteins, Fatty Acids, and Sterols, and Next Generation Sequencing for the Diagnosis and Treatment of Lipid Disorders. En K. R. Feingold, B. Anawalt, A. Boyce, G. Chrousos, W. W. de Herder, K. Dhatariya, K. Dungan, A. Grossman, J. M. Hershman, J. Hofland, S. Kalra, G. Kaltsas, C. Koch, P. Kopp, M. Korbonits, C. S. Kovacs, W. Kuohung, B. Laferrére, E. A. McGee, R. McLachlan, J. E. Morley, M. New, J. Purnell, R. Sahay, F. Singer, C. A. Stratakis, D. L. Trence, D. P. Wilson (Eds.), Endotext. South Dartmouth (MA): MDText.com, Inc. http://www.ncbi.nlm.nih.gov/books/NBK355892/

Shin, S. \& Ajuwon, K. M. (2018). Effects of Diets Differing in Composition of 18-C Fatty Acids on Adipose Tissue Thermogenic Gene Expression in Mice Fed High-Fat Diets. Nutrients, 10(2), E256. DOI: 10.3390/nu10020256 Small, L., Brandon, A. E., Turner, N. \& Cooney, G. J. (2018). 
Modeling insulin resistance in rodents by alterations in diet: What have high-fat and high-calorie diets revealed? American Journal of Physiology. Endocrinology and Metabolism, 314(3), E251-E265. DOI: 10.1152/ ajpendo.00337.2017

Souza Cruz, E. M., Bitencourt de Morais, J. M., Dalto da Rosa, C. V., da Silva Simões, M., Comar, J. F., deAlmeida Chuffa, L. G. \& Seiva, F. R. F. (2020). Long-term sucrose solution consumption causes metabolic alterations and affects hepatic oxidative stress in Wistar rats. Biology Open, 9(3). DOI: 10.1242/bio.047282

Speakman, J. R. (2019). Use of high-fat diets to study rodent obesity as a model of human obesity. International Journal of Obesity (2005), 43(8), 1491-1492. doi: 10.1038/s41366019-0363-7

Stemmer, K., Perez-Tilve, D., Ananthakrishnan, G., Bort, A., Seeley, R. J., Tschöp, M. H., Dietrich, D. R. \& Pfluger, P. T. (2012). High-fat-diet-induced obesity causes an inflammatory and tumor-promoting microenvironment in the rat kidney. Disease models \& mechanisms, 5(5), 627-635. DOI: 10.1242/dmm.009407

Suman, R. K., Ray Mohanty, I., Borde, M. K., Maheshwari, U. \& Deshmukh, Y.A. (2016). Development of an Experimental Model of Diabetes Co-Existing with Metabolic Syndrome in Rats. Advances in Pharmacological Sciences, 2016, 9463476. DOI: 10.1155/2016/9463476

THE POUND MOUSE | Charles River Laboratories. (s/f). https://www.criver.com/products-services/find-model/ pound-mouse?region $=3616$

Third Report of the National Cholesterol Education Program (NCEP) Expert Panel on Detection, Evaluation, and Treatment of High Blood Cholesterol in Adults (Adult Treatment Panel III) Final Report. (2002). Circulation, 106(25), 3143-3143. DOI: 10.1161/circ.106.25.3143

Vague, J. (1956). The degree of masculine differentiation of obesities: A factor determining predisposition to diabetes, atherosclerosis, gout, and uric calculous disease. The
American Journal of Clinical Nutrition, 4(1), 20-34. DOI: 10.1093/ajcn/4.1.20

Velasco, M., Ortiz-Huidobro, R. I., Larqué, C., SánchezZamora, Y. I., Romo-Yáñez, J. \& Hiriart, M. (2020). Sexual dimorphism in insulin resistance in a metabolic syndrome rat model. Endocrine Connections, 9(9), 890-902. DOI: 10.1530/EC-20-0288

Wolfe, R. R., Klein, S., Carraro, F. \& Weber, J. M. (1990). Role of triglyceride-fatty acid cycle in controlling fat metabolism in humans during and after exercise. The American Journal of Physiology, 258(2 Pt 1), E382-389. DOI: 10.1152/ ajpendo.1990.258.2.E382

Wong, S. K., Chin, K.-Y., Suhaimi, F. H., Fairus, A. \& ImaNirwana, S. (2016). Animal models of metabolic syndrome: A review. Nutrition \& Metabolism, 13, 65. DOI: 10.1186/ s12986-016-0123-9

Yang, X.-X., Wang, X., Shi, T.-T., Dong, J.-C., Li, F.-J., Zeng, L.X., Yang, M., Gu, W., Li, J.-P. \& Yu, J. (2019). Mitochondrial dysfunction in high-fat diet-induced nonalcoholic fatty liver disease: The alleviating effect and its mechanism of Polygonatum kingianum. Biomedicine \& Pharmacotherapy = Biomedecine \& Pharmacotherapie, 117, 109083. DOI: 10.1016/j.biopha.2019.109083

Yoon, J.-A., Han, D.-H., Noh, J.-Y., Kim, M.-H., Son, G. H., Kim, K., Kim, C.-J., Pak, Y. K. \& Cho, S. (2012). Meal time shift disturbs circadian rhythmicity along with metabolic and behavioral alterations in mice. PloS One, 7(8), e44053. DOI: 10.1371/journal.pone.0044053

Zhang, X. \& Lerman, L. O. (2016). Investigating the Metabolic Syndrome: Contributions of Swine Models. Toxicologic Pathology, 44(3), 358-366. DOI: 10.1177/0192623316630835

Zhou, Y., Li, W., Zhou, J., Chen, J., Wang, X., Cai, M., Li, F., Xu, W., Carlsson, P.-O. \& Sun, Z. (2019). Lipotoxicity reduces $\beta$ cell survival through islet stellate cell activation regulated by lipid metabolism-related molecules. Experimental Cell Research, 380(1), 1-8. DOI: 10.1016/j.yexcr.2019.04.012 